\title{
Influência dos extrativos na cor e durabilidade natural da madeira de Jurema-preta
}

\author{
Sara Sebastiana Nogueira ${ }^{1}$ Vinicius Gomes Castro@1* Poliana Coqueiro Dias Araújo@1
}

${ }^{1}$ Universidade Federal Rural do Semi-Árido, UFERSA, Av. Francisco Mota, 572, CEP 59625-900, Mossoró, RN, Brasil

\begin{abstract}
Original Article
*Corresponding author: vinicius.castro@ufersa.edu.br

Palavras-chave:

Podridão branca

Colorimetria

Mimosa tenuiflora

Keywords:

White rot

RESUMO: A madeira de Jurema-preta (Mimosa tenuiflora (Willd.) Poiret) é amplamente comercializada na região Nordeste do país, mas nem sempre gera produtos com alto valor agregado devido à desinformação sobre seus potenciais. $\mathrm{O}$ objetivo deste trabalho foi avaliar a influência da variação longitudinal intra espécie na resistência natural ao ataque do fungo Ganoderma sp, causador da podridão branca, e na colorimetria da madeira. A espécie, independente da altura do tronco testada, demonstrou-se altamente resistente ao fungo em ensaio de laboratório. Porém, houve diferença entre a susceptibilidade da madeira do topo do tronco em relação as partes inferiores. Devido a maior presença de alburno, o topo do tronco também apresentou diferença na cor da madeira em relação as demais partes com presença predominante de cerne. $\mathrm{O}$ topo apresentou um tom menos avermelhado do que a da base. As diferenças entre cor e susceptibilidade à podridão branca podem ter sido influenciadas pela maior presença de extrativos solúveis em acetona na madeira do cerne.
\end{abstract}

Colorimetry

Mimosa tenuiflora

Received in

2021/07/07

Accepted on

2021/11/10

Published in

2021/12/30

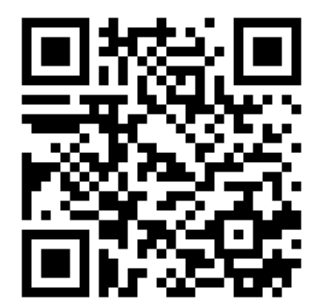

DOI:

https://doi.org/10.34062/afs. v8i4.12728

\section{(cc) BY}

\section{Extractive influence on color and natural durability of Jurema-preta}

\begin{abstract}
Jurema-preta (Mimosa tenuiflora (Willd.) Poiret) wood is largely commercialized in the Northeast region, but not always as products with great added value due to disinformation about its potential. The aim of this work was evaluating the influence of longitudinal variation intra species on the natural resistance to the attack of Ganoderma sp. fungi, responsible for white rot, and on wood colorimetry. The species, no matter the tested high in the stem, showed been highly resistant to the fungi in lab tests. However, there was difference between the top wood susceptibility and lower parts. Due to the higher presence of sapwood, the top wood also showed wood color difference compared with the other parts with predominant presence of heartwood. The top showed less red hue than the bottom part. The differences between color and white rot susceptibility could be influenced by the higher presence of acetone soluble extractives in the heartwood.
\end{abstract}




\section{Introdução}

A Jurema-preta (Mimosa tenuiflora (Willd.) Poiret) é uma espécie nativa do bioma Caatinga da família Fabaceae. Sua madeira apresenta cerne e alburno distintos, sendo o primeiro de cor marrom e o segundo de um tom amarelo claro acinzentado (Morais et al. 2018). É uma espécie importante para a região Nordeste por apresentar grande potencial madeireiro e por ser pioneira, capaz de formar florestas em diversas áreas degradas com solo exposto ou erodido (Ferreira et al. 2020). Seu potencial madeireiro pode ser confirmado pelo fato de, por exemplo, no estado do Rio Grande do Norte, a madeira do gênero Mimosa é a nativa mais transportada em volume. Sua utilização é em grande parte voltada para produção de lenha para uso doméstico, comercial e industrial, principalmente a indústria de cerâmica (Sousa Júnior et al. 2021).

Contudo, a madeira de Jurema possui um potencial além do energético. Ela também é usada como estacas, moirões e em outras atividades laborais (Sousa et al. 2018), em construções domésticas (Lima et al. 2018), como matéria prima para indústria moveleira (Rocha et al. 2015) ou indústria de painéis de madeira (Nascimento et al. 2013). E o potencial de uso dessa espécie não se baseia apenas nas propriedades físico-mecânicas de sua madeira. É sabido que seus extrativos fenólicos possuem capacidade antimicrobiana e antioxidantes que podem ser utilizadas em indústrias de embalagens biodegradáveis (Silva et al. 2021).

A presença de extrativos é tida como a causa de inibição do crescimento dos fungos Ganoderma, capazes de causar podridão em diversas espécies tropicais (Paula e Brioso, 2021). Fungos deste gênero são cosmopolitas e causam podridão branca em raízes e partes inferiores do tronco. Por isso é um grande risco para produtos madeireiros que terão contato com o solo, como por exemplo mourões. O nível de agressividade do ataque está correlacionado não só a espécie do fungo, mas também ao tipo da madeira e sua química (Loyd et al. 2018). Paes et al. (2015) indicaram o potencial da madeira de Juremapreta devido a sua alta durabilidade natural. Os autores avaliaram a resistência de sete espécies nativas da Caatinga a ataques de fungos e cupins xilófagos e classificaram a Jurema-preta como uma das duas e espécies mais resistentes aos organismos testados.

Os tipos de extrativos presentes em uma madeira também influenciam diretamente sua cor (Maia et al. 2019). Quando se considera explorar o potencial madeireiro de uma espécie, a compreensão da cor torna-se essencial no processo. A cor de um produto é o gatilho de compra do consumidor por estar ligada a percepção sensorial e emotiva (Cheng et al. 2019). No caso de espécies como a Juremapreta, que possuem cerne e alburno distintos, é importante que a cor seja quantificada para se prever variações em peças longitudinais que pode afetar a aparência final dos produtos.

Diante disto, o objetivo deste trabalho foi avaliar a variação da composição química ao longo do troco da espécie Jurema-preta e seus efeitos na resistência à podridão branca e aos parâmetros colorimétricos da madeira.

\section{Material e Métodos}

Três indivíduos da espécie Jurema-preta (Mimosa tenuiflora (Willd.) Poiret), com diâmetro à altura do peito (DAP) médio de $12 \mathrm{~cm}$, foram coletados em uma área sob manejo florestal localizado no município de Upanema/RN. Os troncos foram seccionados em três toretes relativos às alturas de $0 \%$ (base), 50\% (meio), e 100\% (topo), referentes à altura dos indivíduos que variou entre 5 e 6 metros.

Cada torete foi desdobrado em uma serra circular para gerar pequenas tábuas radiais com as dimensões de aproximadamente $5 \times 2 \times 20 \mathrm{~cm}$ de largura, espessura e comprimento, respectivamente. A madeira residual da produção dos corpos de prova foi reaproveitada para análise das propriedades químicas. O resíduo foi moído em um moinho de facas tipo Wiley, para cada altura separadamente. O material foi então peneirado, sendo utilizado o passante em peneira de abertura de 40 mesh (TAPPI, 2012). Para se evitar entupimento do papel de filtro utilizado no processo, a norma de preparação da amostragem foi adaptada sendo descartado o material fino passante na peneira de 100 mesh. Foram feitas três repetições para cada altura na determinação do teor de extrativo solúveis em água fria, água quente (TAPPI, 2008) e em acetona (TAPPI, 2007).

Para avaliar a variação da cor ao longo do tronco da Jurema-preta, foram realizadas análises colorimétricas com cinco leituras no sentido radial de cada tábua. Nas tábuas oriundas da base e meio dos troncos, as medidas foram realizadas na parte referente ao cerne. Nestas amostras, o cerne representava cerca de $90 \%$ da área superficial. Porém, nos indivíduos produzidos do topo do tronco, as medições foram feitas em madeira de alburno, uma vez que o material ainda não havia sofrido o processo de cernificação. As análises foram realizadas utilizado o colorímetro Konica Minolta CR-410, por meio do sistema CIE-L*a*b*. Avaliouse os parâmetros colorimétricos: L (luminosidade), $a^{*}$ (coordenada no eixo vermelho-verde) e $b^{*}$ (coordenada do eixo azul-amarelo). Utilizou-se as equações (1) e (2) para calcular os parâmetros C (saturação) e h*(ângulo de tinta): 


$$
\begin{aligned}
& \mathrm{C}=\frac{(a * 2+b * 2)}{2} \\
& \mathrm{~h} *=\tan -1\left(\frac{\mathrm{b} *}{\mathrm{a} *}\right)
\end{aligned}
$$

Em que: $\mathrm{a}^{*}=$ coordenada no eixo vermelhoverde; $b^{*}=$ coordenada no eixo azul-amarelo; $\mathrm{C}=$ Saturação; $h^{*}=$ ângulo de tinta

Para a avaliação da resistência da madeira ao ataque de podridão branca, o fungo utilizado foi o Ganoderma sp. coletado em ambiente natural de Caatinga. $\mathrm{O}$ material foi isolado e repicado em meio de cultura BDA (batata-dextrose-ágar) e mantidos em estufa incubadora de demanda bioquímica de oxigênio (BOD) a $28^{\circ} \mathrm{c}$ de temperatura, com fotoperíodo de $12 \mathrm{~h}$.

Para cada altura avaliada, foram produzidos 20 corpos de prova com as dimensões de 2,5 x 2,5 x $1 \mathrm{~cm}$ de sentido tangencial, radial e longitudinal, respectivamente. Sendo para cada tratamento, 10 corpos de prova foram inoculados com o fungo, os demais foram mantidos como testemunha.

$O$ ensaio de deterioração da madeira foi conduzido segundo a norma ASTM D 2017 (ASTM, 2005). Frascos de vidros com tampa metálica rosqueável foram preenchidos com $40 \mathrm{~g}$ de solo cada, em sequência procedeu-se a esterilização utilizando tratamento térmico por meio de autoclavagem a 120 ${ }^{\circ} \mathrm{c}$ por 30 minutos. O solo utilizado foi do tipo Cambissolo, com pH 6,7, coletados no município de Afonso Bezerra/RN. Em câmara de fluxo laminar foi adicionado $9 \mathrm{ml}$ de água destilada e autoclavada a cada frasco, propiciando um meio adequado para desenvolvimento do microrganismo. O fungo foi então inoculado, para cada amostra foi utilizado dois discos de meio de cultura, completamente cobertos pelos micélios do microrganismo, com $10 \mathrm{~mm}$ de diâmetro. $\mathrm{O}$ material foi mantido na BOD a $28^{\circ} \mathrm{c}$ de temperatura, com fotoperíodo de $12 \mathrm{~h}$ e avaliado após 12 semanas.

A avaliação do índice de deterioração foi feita através do cálculo de perda de massa seca indicado na equação (3). Com base neste valor, as amostras foram classificadas de acordo com sua resistência natural à podridão branca (Tabela 1) (ASTM, 2005).

$$
\mathrm{PM}=\frac{(m i-m f)}{m f} * 100
$$

Em que: $\mathrm{PM}=$ perda de $\operatorname{massa}(\%) ; \mathrm{mi}=$ massa seca inicial $(\mathrm{g}) ; \mathrm{mf}=$ massa seca final $(\mathrm{g})$.

Para avaliar o grau de degradação dos polissacarídeos da madeira pelo fungo, foi avaliada a solubilidade das amostras em hidróxido de sódio a $1 \%$ (TAPPI, 2002) antes e depois do ensaio de deterioração.
Tabela 1. Classificação da resistência da madeira ao ataque de fungos indicada pela ASTM (2005)

\begin{tabular}{cc}
\hline Perda de massa (\%) & $\begin{array}{c}\text { Categoria de } \\
\text { resistência ao fungo } \\
\text { testado }\end{array}$ \\
\hline 0 a 10 & Altamente resistente \\
1 a 24 & Resistente \\
25 a 44 & $\begin{array}{c}\text { Moderadamente } \\
\text { resistênte } \\
\geq 45\end{array}$ \\
& Pouco ou não \\
& resistente \\
\hline
\end{tabular}

Realizou-se a Análise de Variância (ANOVA) com Delineamento Inteiramente Casualizado (DIC) para as variáveis de propriedades químicas e parâmetros colorimétricos da madeira de Jurema-preta a diferentes alturas. Além disto, avaliou-se ainda a perda de massa da madeira, a solubilidade em hidróxido de sódio a $1 \%$ antes e após o ensaio com fungo de podridão branca por meio de análise de variância (ANOVA) em arranjo fatorial para verificar a interação entre dois fatores: altura e ataque. Para tabulação dos dados utilizou-se o programa estatístico Sisvar 5.6 e, após normalidade caracterizada pelos testes Skewness e Kurtosis, aplicou-se o teste de Tukey com um nível de significância a 5\% de probabilidade.

\section{Resultados}

Não houve diferença estatística entre os valores médios de teores de extrativos solúveis em água fria da madeira de Jurema-preta em diferentes posições do tronco (tabela 2). Porém, quando usado água quente, a quantidade extraída foi estatisticamente inferior na base do que no resto da árvore. Já para os extrativos apolares solúveis em acetona, o topo apresentou menor quantidade do que as partes inferiores do tronco.

A madeira de Jurema-preta é mais escura no topo do que no restante do tronco (tabela 3). Porém, segundo Camargos e Gonçalez (2001), apenas madeiras com luminosidade acima de 56 podem ser consideradas claras, desta forma a Jurema-preta é uma madeira de tons escuros. O topo da madeira também diferiu estatisticamente das demais partes quanto ao critério de pigmentação avermelhada e ângulo de tinta.

$\mathrm{O}$ ângulo de tinta para madeiras, varia de 0 a $90^{\circ}$, sendo que valores mais altos indicam tonalidades mais amareladas, enquanto valores mais baixos tendem ao avermelhado (Maia et al. 2020). A combinação menos avermelhadas do que as das partes inferiores dos resultados de $\mathrm{a}^{*} \mathrm{e} \mathrm{h} *$ indicaram que o topo da árvore apresenta madeiras. 
Tabela 2. Teores de extrativos em diferentes solubilidades para madeira de Jurema-preta ao longo do troco.

\begin{tabular}{lccc}
\hline Alturas & \multicolumn{3}{c}{ Teor de Extrativos $(\%)$} \\
\cline { 2 - 4 } & Água fria & Água quente & Acetona \\
\hline Base & $4,84(8,01) \mathrm{a}$ & $2,07(13,63) \mathrm{a}$ & $11,33(3,69) \mathrm{b}$ \\
Meio & $5,62(7,02) \mathrm{a}$ & $4,61(7,38) \mathrm{b}$ & $9,33(1,31) \mathrm{b}$ \\
Topo & $4,96(2,16) \mathrm{a}$ & $3,88(18,27) \mathrm{b}$ & $5,27(7,81) \mathrm{a}$ \\
\hline
\end{tabular}

Em que: Valores seguidos de uma mesma letra, em uma mesma coluna, não diferem estatisticamente. Valores em parênteses referentes ao coeficiente de variação $(\%)$.

Tabela 3. Parâmetros colorimétricos da madeira de Jurema-preta em três posições diferentes do tronco.

\begin{tabular}{cccccc}
\hline Alturas & $\mathbf{L}$ & $\mathbf{a}^{*}$ & $\mathbf{b}^{*}$ & $\mathbf{C}$ & $\mathbf{h}^{*}$ \\
\hline Base & $45,47(6,77)$ a & $12,71(4,63) \mathrm{b}$ & $22,09(12,71)$ a & $25,54(9,58)$ a & $59,79(5,85) \mathrm{a}$ \\
Meio & $47,75(6,21)$ a & $11,97(5,19) \mathrm{b}$ & $22,18(15,46)$ a & $25,26(12,19)$ a & $61,17(7,26)$ a \\
Topo & $51,73(7,27) \mathrm{b}$ & $9,83(17,72)$ a & $23,06(10,26)$ a & $25,16(7,70)$ a & $66,69(7,41) \mathrm{b}$ \\
\hline
\end{tabular}

Em que: $\mathrm{L}=$ Luminosidade; $\mathrm{a}^{*}=$ pigmento vermelho-verde; $\mathrm{b}^{*}=$ pigmento azul-amarelo; $\mathrm{C}=$ cromaticidade; $\mathrm{h}=$ ângulo de tinta; Valores seguidos de uma mesma letra, em uma mesma coluna, não diferem estatisticamente. Valores em parênteses referentes ao coeficiente de variação $(\%)$.

Tabela 4. Perda de massa de madeiras de Jurema-preta atacada por podridão branca

\begin{tabular}{ccc}
\hline Alturas & \multicolumn{2}{c}{ Perda de massa (\%) } \\
\cline { 2 - 3 } & Atacado & Testemunha \\
\hline Base & $1,16(30,36) \mathrm{aA}$ & $0,80(25,73) \mathrm{aA}$ \\
Meio & $1,63(30,69) \mathrm{aA}$ & $1,09(26,25) \mathrm{aA}$ \\
Topo & $4,78(50,79) \mathrm{bB}$ & $0,68(50,89) \mathrm{aA}$ \\
\hline
\end{tabular}

Em que: Valores seguidos de uma mesma letra minúscula, em uma mesma coluna, não diferem estatisticamente. Valores seguidos de uma mesma letra maiúscula, em uma mesma linha, não diferem estatisticamente. Valores em parênteses referentes ao coeficiente de variação $(\%)$.

Após ensaio de laboratório de deterioração pelo fungo causador da podridão branca, apenas a madeira do topo da Jurema-preta apresentou valores estatisticamente diferentes da madeira que não foi inoculada (Tabela 4). E mesmo sendo mais susceptível do que as partes inferiores do tronco, o topo apresentou perda de massa inferior a $10 \%$, sendo então uma madeira classificada como altamente resistente de acordo com a ASTM (2005).

Tabela 5. Solubilidade em $\mathrm{NaOH} 1 \%$ para madeiras de Jurema-preta com e sem ataque de podridão branca

\begin{tabular}{|c|c|c|}
\hline \multirow[t]{2}{*}{ Alturas } & \multicolumn{2}{|c|}{ Solubilidade em NaOH $1 \%$} \\
\hline & Atacada & Testemunha \\
\hline Base & $\begin{array}{c}26,29(1,15) \\
\mathrm{aA}\end{array}$ & $24,83(10,44) \mathrm{aA}$ \\
\hline Meio & $\begin{array}{c}24,27(8,41) \\
\mathrm{aA}\end{array}$ & $23,20(12,69) \mathrm{aA}$ \\
\hline Topo & $\begin{array}{c}23,74(12,20) \\
\mathrm{aA}\end{array}$ & $22,78(7,33)$ aA \\
\hline
\end{tabular}

Em que: Valores seguidos de uma mesma letra minúscula, em uma mesma coluna, não diferem estatisticamente. Valores seguidos de uma mesma letra maiúscula, em uma mesma linha, não diferem estatisticamente. Valores em parênteses referentes ao coeficiente de variação (\%).

O teste de solubilidade em hidróxido de sódio $1 \%$ avalia também a quantidade de polissacarídeos de baixo peso molecular (TAPPI, 2002). Quando há ataque de fungos de podridão, que possuem capacidade de degradar e reduzir o peso molecular da celulose, a solubilidade da madeira tende a aumentar. No caso da Jurema-preta, não houve diferença estatística entre os valores, sejam ao longo do tronco, ou entre madeiras sadias e atacadas, que confirmou a resistência da espécie ao ataque do Ganoderma sp. (tabela 5).

\section{Discussão}

Brito et al. (2014) também haviam avaliado a diferença dos teores extrativos da Jurema-preta ao longo do fuste. Diferente dos resultados encontrados neste trabalho, os autores reportaram que não houve diferença estatística entre os valores de extrativos totais entre a base e o topo. Porém, é importante ressaltar que no trabalho citado não houve diferenciação entre os extrativos polares e apolares.

Os teores de extrativos variam ao longo do tronco não somente em quantidade, mas também em composição. Por exemplo, no topo do tronco, há uma maior quantidade de células vivas (alburno) que resulta em maiores teores de glicerídeos e amido (substâncias de reserva) do que no cerne, presente em maior proporção na base do tronco (Jansson e Nilvebrant, 2009). De acordo com a norma TAPPI (2008), o amido é solúvel em água quente. Desta forma, justifica-se a média estatisticamente superior 
do teor de extrativos em água quente da madeira de topo em relação às demais.

De acordo com Brémaud et al. (2011), o tipo de solvente usado também pode indicar a localização dos extrativos na madeira. Acetona e água quente, devida a suas polaridades, podem inchar a parede celular e alcançar compostos localizados em seu interior, além dos extrativos presentes no lúmen.

Paes et al. (2013) indicaram um teor de extrativo apolar para a Jurema-preta de $9,96 \%$, valor este semelhante ao encontrado na porção central do tronco neste trabalho. A explicação para maior quantidade de extrativos apolares nas porções inferiores da árvore também se baseia na diferente porcentagem de cerne. Durante o processo de cernificação, a árvore produz extrativos para proteger a madeira de ataques de biodeterioradores. Substâncias fenólicas possuem capacidade fungicida e geralmente são encontradas apenas no cerne. Embora sejam solúveis em água, são encontradas em extrações feitas com solvente acetona (Jansson e Nilvebrant, 2009).

Maia et al. (2019) encontraram uma correlação entre extrativos polifenólicos solúveis em acetona e o parâmetro colorimétrico $a^{*}$ e, consequentemente, $\mathrm{h} *$. Para a espécie Jurema-preta, essa tendência também foi observada. De acordo com Melo et al. (2019), os valores colorimétricos encontrados para a Jurema-preta, mesmo com variação de cor entre alburno e cerne, poderiam a classificar como uma madeira rosa acinzentada.

Lukmandaru e Sayudha (2017) correlacionaram os parâmetros $\mathrm{L}$ e b* com a porção polar dos extrativos. A Jurema-preta apresentou uma baixa concentração deste tipo de extrativo, o que justificaria a falta de influência na tonalidade amarela. O efeito dessa correlação foi observado apenas no fato do topo ser uma madeira ligeiramente mais clara do que as mais ricas em cerne.

Os valores médios de cromaticidade foram considerados alto sendo acima de 23,39 (Camargos e Gonzalez 2001). Essa alta saturação das cores também foi observada em madeiras do mesmo gênero Sabiá (Mimosa caesalpiniifolia Benth) (Maia et al. 2019).

Em relação a resistência natural da Juremapreta em diferentes posições do tronco, Paes et al. (2015) também classificaram a espécie como muito resistente com valores de perda abaixo de $2 \%$. Neste trabalho, o topo apresentou maior susceptibilidade, provavelmente devido a quantidade de madeira juvenil, mas que não chegou a afetar sua classificação de resistência.

A alta resistência ao apodrecimento pode estar ligada a composição química do extrativo. Os extrativos da espécie Jurema-preta são naturalmente ricos em compostos fenólicos totais, sendo estes produzidos como mecanismo de defesa do vegetal quando submetido a condições de estresse fisiológico (Silva et al. 2021). Uma vez que as espécies estudadas foram nativas da região do semiárido, pode-se considerar que o estresse hídrico funcione como gatilho na produção deste componente químico que reflete na durabilidade natural da madeira.

A extração com $\mathrm{NaOH} 1 \%$ consiste em ácidos graxos, oléicos e linólicos, mas além disso, também tem capacidade de dissolver a maior parte das hemiceluloses (Rosamah et al. 2020). Fungos causadores de podridão são capazes de degradar a celulose, reduzindo seu peso molecular, tornando-a solúvel ao hidróxido de sódio. Porém, a Juremapreta submetida ao fungo não apresentou diferença nos valores médios de solubilidade. O mecanismo de ataque do fungo Ganoderma sp. envolve a degradação inicial da lignina pela produção de peróxido de magnésio (Schmidt 2006).

\section{Conclusões}

Existe uma variação na quantidade dos teores de extrativos intra espécie para a madeira de Juremapreta (Mimosa tenuiflora). A maior quantidade de extrativos solúveis em acetona no cerne, predominante nas madeiras da região da base do troco da árvore, influenciou na cor avermelhada e na durabilidade natural da madeira.

Contudo, mesmo a madeira do topo, formada apenas de alburno, apresentou resultados que permitem classificar a madeira da espécie como altamente resistente ao ataque do fungo Ganoderma sp. causador de podridão branca.

\section{Referências}

ASTM. American Society for Testing and Materials (2005) ASTM D-2017. Standard method for accelerated laboratory test of natural decay resistance for woods. Philadelphia: ASTM. 2005

Brémaud I, Amusant N, Minato K., Gril, J, Thibaut B (2011) Effect of extractives on vibrational properties of African Padauk (Pterocarpus soyauxii Taub.). Wood Science Technology, 45(3): 461- 472. doi 10.1007/s00226-010-0337-3.

Brito RF, Stangerlin DM, Calegari L, Pariz E, Calegari CCA, Gatto DA (2014) Influência da variação axial, radial e granulométrica da serragem na composição química da madeira de jurema-preta. Ciência da Madeira, 5(2):111-117. doi: 10.12953/2177-6830.v05n02a05

Camargos JAA, Gonçalez JC (2001) Colorimetria aplicada como instrumento na elaboração de uma tabela de cores de madeira. Brasil Florestal, 20(71):30-41.

Cheng FF, Wu CS, Leiner B (2019). The influence of user interface design on consumer perceptions: a 
cross-cultural comparison. Computer in Human Behavior, 101(1): 394-401. doi: 10.1016/j.chb.2018.08.015.

Ferreira MB, Bakke OA, Souse GG, Bakke IA, Azevedo SRV, Ferreira WC (2020) Spatial distribution, regeneration, growth and thicket formation of thornless Mimosa tenuiflora in a Caatinga site of Northeast Brazil. Journal of Experimental Agriculture International, 43(3): 137149. doi: 10.9734/JEAI/2020/v42i330491

Jansson MB, Nilverbrant NO (2009) Wood extractives In: Ek M, Gellerstedt G, Henriksson G. Pulp and Paper Chemistry and Technology. Volume 1. Berlin: De Gruyter. p.147-171

Lima JRF, Lima GDS, Lucena CM, Carvalho TKN, Lucena RFP (2018) Inventário in situ como método para avaliação da extração de recursos madeireiros na Caatinga: estudo de caso no município de cabeceiras (Paraíba, Brasil). Revista Nordestina de Biologia, 26(1):1-18. doi: 10.22478/ufpb.22361480.2018v26n1.46051

Loyd AL, Held BW, Linder E., Smith JA, Blanchette RA (2018) Elucidating wood decomposition by four species of Ganoderma from the United States. Fungal biology, 122(4), 254-263. doi: 10.1016/j.funbio.2018.01.006

Lukmandaru G, Sayudha IGND (2017). Colour properties and extractive content in young teak woods. In: 9th International Symposium of Indonesian Wood Research Society, Denpasar, Indonésia. p.164-174.

Maia JH, Ferreira LMM, Castro VG (2020) Influence of extractives on the color of woods from Caatinga. Advances in Forestry Science, 7(2):10431048. doi: 10.34062/afs.v7i2.9421

Melo RR, Stangerlin DM, Del Menezzi CHS, Castro VG, Souza MJC, Medeiros DT (2019) Grouping of commercial tropical timber using wood color. Revista de Ciências Agrárias, 62:1-6. doi: 10.22491/rca.2019.310

Morais RM, Cunha MCL, Santana GM, Paes JB (2018) Dendrological characterization as inspection resources of Caatinga wood market. Floresta $e$ Ambiente, 25(3):e20170813. doi: 10.1590/21798087.081317

Nascimento MF, Christoforo AL, Bertolini MS, Chahud E, Lahr FAR (2013) Painéis de partículas homogêneas fabricados com espécies de manejo da região da caatinga do Brasil. Construindo, 5(2):1-10
Paes JB, Melo RR, Lima CRD (2015) Resistência natural de sete madeiras a fungos e cupins xilófagos em condições de laboratório. Cerne, 13(2):160-169.

Paes JB, Lima CRD, Oliveira ED, Medeiros Neto PND (2013) Características físico-química, energética e dimensões das fibras de três espécies florestais do semiárido brasileiro. Floresta $e$ Ambiente, 20 (4): 550-550. doi: 10.4322/ floram.2013.022

Paula TC, Brioso PST (2021) Resistência de espécies arbóreas tropicais à ação de Ganoderma philippii. Ciência Florestal, 31(1):393-416. doi: 10.5902/1980509843747

Rocha HLS, Paes JB, Miná AJS, Oliveira E (2015) Caracterização física mecânica da madeira de jurema-preta (Mimosa tenuiflora) visando seu emprego na indústria moveleira. Agrária, 10(2):262267. doi: 10.5039/agraria.v10i2a3772

Rosamah E, Ferliyantu F, Kuspradini H, Dungani R, Aditiawati P (2020) Chemical contente in two teak wood (Tectona grandis Linn. F.) that has been used for 2 and 60 years. Bio Journal of Biological Science, Technology and Management, 2(1): 15-19. doi: 10.5614/3bio.2020.2.1.3

Schmidt O (2006) Wood and tree fungi: biology, damage, protection, and use. Berlim: Springer. 334p.

Silva IDL, Oliveira FSM, Andrade MF (2021) Avaliação das potencialidades dos extratos vegetais de jurema preta (Mimosa tenuiflora) e cajueiro (Anacardium occidentale L.) para uso em embalagens ativas antimicrobianas e antioxidantes. Matéria, 26(1):e12924. doi: 10.1590/S1517707620210001.1224

Souza AP, Costa FCP, Alencar RF, Lima SFB (2018) Exploração e utilidade do potencial madeireiro da Caatinga no município de Aurora estado do Ceará. Pesquisa e Ensino em Ciências Exatas e da Natureza, 2(2):158-168. doi: 10.29215/pecen.v2i2

Sousa Júnior AD, Canto JL, Costa WPLB (2021) Produtos florestais nativos, legalizados e comercializados no Rio Grande do Norte. Cadernos de Ciência \& Tecnologia, 38(1):e26779. doi: 10.35977/0104-1096.cct2021.v38.26779

TAPPI. Technical Association of the Pulp and Paper Industry (2007). TAPPI T $204 \mathrm{~cm}$-97. Solvent extractives of wood and pulp. Atlanta: TAPPI Press.

TAPPI. Technical Association of the Pulp and Paper Industry (2008) TAPPI T $207 \mathrm{~cm}$-99. Water solubility of wood and pulp. Atlanta: TAPPI Press. 
Nogueira et al.

TAPPI - Technical Association of the Pulp and Paper Industry (2002) TAPPI T $212 \mathrm{om}-02$. One percent sodium hydroxide solubility of wood and pulp. Atlanta: TAPPI Press.

TAPPI - Technical Association of the Pulp and Paper Industry (2012) TAPPI T $257 \mathrm{~cm}$-12. Sampling and preparing wood for analysis. Atlanta: TAPPI Press. 